\title{
THE STRUCTURE OF THE ÆTHER.
}

BY DR. H. BATEMAN.

(Read before the American Mathematical Society, February 28, 1914.)

\section{§1. Introduction.}

IN Maxwell's electromagnetic theory of light the broad view is taken that waves of light are manifestations of certain electromagnetic phenomena in the æther which are included in the general class of processes capable of being described by means of the partial differential equations

$$
\operatorname{rot} M=-\frac{i}{c} \frac{\partial M}{\partial t}, \quad \operatorname{div} M=0 .
$$

In these equations $M$ denotes the complex vector $H+i E$, the vectors $E$ and $H$ represent the electric and magnetic intensities respectively, $c$ is a constant which is usually called the velocity of light, and $\operatorname{rot} M$ denotes the complex vector whose components are of type

$$
\frac{\partial M_{z}}{\partial y}-\frac{\partial M_{y}}{\partial z}
$$

when right-handed rectangular axes are used.

In the extensions of the theory which have been made by H. A. Lorentz* and Sir Joseph Larmor, $\dagger$ it is recognized that the fundamental equations (1) must not be regarded as holding for all real values of the variables $x, y, z, t$; the exceptional domains or singular points are, moreover, regarded as instrumental in the production of waves or disturbances in the æther.

The hypothesis generally adopted by Lorentz is that there are exceptional domains, which, when viewed at any instant, consist of an enormous aggregate of small closed regions. These domains are supposed to be occupied by electricity

* Archives néerlandaises, vol. 25 (1892); Amsterdam Proceedings (1902), p. 305 .

$\dagger$ Phil. Trans. A, vol. 185 (1894), p. 719; vol. 186 (1895), p. 695; Atther and Matter, Cambridge (1900). 
whose volume density $\rho$ is generally a function of $x, y, z, t$. The motion of the electricity is supposed to be specified by a vector function $v$.

The vector functions $E$ and $H$ are defined for all real values of $x, y z, t$ with the aid of the retarded potentials of L. Lorenz. These potentials $A$ and $\Phi$ are expressed as triple integrals involving $\rho$ and $v$ and the vectors $E, H$ are derived from them with the aid of the relations

$$
E=-\frac{1}{c} \frac{\partial A}{\partial t}-\frac{\partial \Phi}{\partial x}, \quad H=\operatorname{rot} A .
$$

When $E$ and $H$ are defined in this way they are found to satisfy equations (1) when the space-time point $(x, y, z, t)$ does not belong to the exceptional domains: for points in the regions occupied by electricity, the modified equations

$$
\operatorname{rot} M=\rho v-\frac{i}{c} \frac{\partial M}{\partial t}, \quad \operatorname{div} M=i \rho
$$

are satisfied.

Starting from these equations Lorentz and Larmor have obtained, by a process of averaging, a set of equations capable of describing the principal optical and electromagnetic properties of a material medium. These more complicated equations are usually called the macroscopic equations, and equations (3) the microscopic equations or fundamental equations of the theory of electrons.

Sir Joseph Larmor has used a more fundamental theory* in which the simple equations (1) are assumed to be the whole scheme and electricity is regarded as consisting of so-called isolated point singularities in the æther, that is, points at which $E$ and $H$ become infinite in a certain manner. A solution of equations (1) which can be regarded as giving an exact specification of an elementary electromagnetic field with a single isolated point singularity of the required type has been obtained by A. Liénard † for the case in which the velocity of the singularity is always less than that of light. It turns out that the electric charge associated with the singularity does not vary with the time. With the aid of this solution, which was originally derived from the retarded

* Fither and Matter, p. 77.

$\dagger L^{\prime}$ Eclairage électrique, t. 16 (1898), pp. 5, 53, 106. 
potentials for a volume distribution of electricity, it is possible to reverse matters and effect a transition from equations (1) to equations (3) by a process of averaging or superposition. The exceptional domains within which $\rho$ and $\rho v$ are different from zero are now supposed to be occupied by a closely packed aggregate of the point singularities of the superposed elementary electromagnetic fields.

One advantage of this more fundamental theory is that it emphasizes two properties of electricity, namely the invariance of the electric charge and the limitation to which its velocity of motion is subject.* The mathematical discussion of the theory is at present incomplete, partly because some hypothesis is needed which will reveal the structure of the æther and show clearly that electric charges are indeed point singularities in the medium. In this note an hypothesis is considered which fulfils some of the requirements; the mathematical analysis is, however, still incomplete.

\section{§ 2. Vector Fields with Moving Singular Curves.}

I have shown elsewhere that there are vector fields satisfying equations (1) in which the vector functions $E, H$ become infinite at points lying on certain moving curves. There is, moreover, at present no evidence that these fields can be built up by superposition from elementary electromagnetic fields of Liénard's type. I propose, therefore, to call them athereal fields so as to distinguish them from the electromagnetic fields which possess the property just mentioned. Whether æthereal fields have a physical existence or not is an open question, but I propose to consider the hypothesis that when a large number of æthereal fields are superposed their singular curves indicate the structure of an "æther" which is capable of supporting a certain type of electromagnetic field which is mathematically determined by the properties of the superposed æthereal fields.

According to this view the structure of the æther depends

*This limitation was first clearly pointed out by J. J. Thomson, Recent Researches (1893), p. 21. See also O. Heaviside, Phil. Mag., vol. 27 (1889), p. 324.

$\dagger$ Philosophical Magazine, Oct., 1913, Jan., 1914; Annals of Mathematics, 1914; The Mathematical Analysis of Electrical and Optical Wave Motion on the Basis of Maxwell's Equations, Cambr. Univ. Press, 1914, Ch. 8. This will be denoted hereafter by $E$. 
to some extent upon the type of electromagnetic field which it supports, and vice versa. There must consequently be some definite mathematical relation between the electromagnetic field and the superposed æthereal fields whose singular curves indicate the structure of the æther. We shall now consider a relation which is suitable for the case in which the electromagnetic field is an elementary field of Liénard's type.

\section{§3. Conjugate Fields.}

Two vector fields in which the complex vectors are $M, M^{\prime}$ respectively are said to be conjugate when the scalar product $\left(M M^{\prime}\right)$ is zero. A field which is conjugate to itself is said to be self-conjugate and in this case $\left(M^{2}\right)=0$.

If the direction of the flow of energy in two fields is the same at each point and both fields are self-conjugate they are also conjugate to one another. To prove this we remark that since the direction of the flow of energy is at right angles to both the electric and magnetic intensities, the vectors $E, H, E^{\prime}, H^{\prime}$ associated with any point must lie in one plane. Moreover, since both fields are self-conjugate the electric and magnetic intensities in each field are perpendicular, hence we must have $M^{\prime}=k M$, where $k$ is a complex quantity. It is now clear that the relation $\left(M M^{\prime}\right)=0$ is a consequence of the two relations $\left(M^{2}\right)=0,\left(M^{2}\right)=0$. It should be noticed that the present argument fails if the flow of energy takes place in opposite directions in the two fields, because then the correct conclusion is that $M^{\prime}=k M^{*}$ where

$$
M^{*}=H-i E .
$$

Let us now consider an electromagnetic field and a large number of mutually conjugate self-conjugate æthereal fields each of which is conjugate to the electromagnetic field. We shall regard the singular curves of these æthereal fields as forming an element of the other and shall suppose that it is this element which supports the particular electromagnetic field in question. In the example of an element which will be given presently the singular curves meet in a point which is the point singularity of the electromagnetic field supported by the element.

It should be remarked that the condition of conjugacy is invariant when a transformation of variables is made which 
leaves equations (1) unaltered in form. Our hypothesis is thus compatible with the modern ideas of relativity. It should also be noticed that when a number of mutually conjugate fields are superposed the term of type $\left(E^{2}\right)-\left(H^{2}\right)$ in the Lagrangian function of the total field is the sum of the terms of this type belonging to the different constituent fields. It is possible then that the condition of conjugacy has a dynamical significance. The fields used in forming an element of the æther can be regarded as analogous to the terms of a Fourier series or the elements of a Fourier integral; each constituent field is supposed to be multiplied by a factor or coefficient, and by giving different values to these coefficients different states of vibration of the fibers of the element may be represented mathematically. With regard to the nature of this vibration or disturbance which can run along a singular curve or fiber the only certain information we are able to give at present is that its orientation may vary with the time and be different for different fibers.

It is by no means' certain that the fibers of an element should be reggarded as filling the whole of space. If we consider only those æthereal fields whose singular curves lie within a kind of tube or cone having its vertex at the point singularity of the electromagnetic field, it appears likely, judging from some simple cases which have been considered,* that it is possible to choose the coefficients so that when both the electromagnetic and æthereal fields are superposed the vectors $E, H$ in the total field are zero outside the tube. This corresponds to the type of element which has been considered by Sir Joseph Thomson in his theory of the structure of the electric field. $\dagger$

\section{§4. A Solution of the Fundamental Equations.}

The components of a vector $M$ which satisfies the equations (1) are known to be solutions of the wave equation

$$
\frac{\partial^{2} u}{\partial x^{2}}+\frac{\partial^{2} u}{\partial y^{2}}+\frac{\partial^{2} u}{\partial z^{2}}=\frac{1}{c^{2}} \frac{\partial^{2} u}{\partial t^{2}} .
$$

The type of singular curve which is typical of an æthereal

* See for instance $E$, p. 121.

† Recent Researches, Ch. I; Phil. Mag., vol. 19 (1910), p. 301; Ibid., Oct., 1913, Dec., 1913. See also N. R. Campbell, The New Quarterly, 1909; D. N. Mallik, Phil. Mag., vol. 26 (1913), p. 144. 
field is obtained by considering the case in which the components of $M$ are all wave functions of type $u=\gamma f(\beta)$, where the function $f$ is different for each component but the functions $\beta$ and $\gamma$ are the same. The quantities $\beta$ and $\gamma$ are supposed to be functions of $x, y, z, t$; an essential property of $\beta$ is that it must be complex so that the equation $1 / f=0$ may be equivalent to two real relations connecting $x, y, z, t$ and so generally represent a moving curve. It has been shown that the velocity of this singular curve in a direction at right angles to the tangent at any point is never greater than the velocity of light.*

The problem of finding all solutions of the wave equation having the general form $\gamma f(\beta)$ has not been solved completely, but in the particular case in which this expression can be regarded as included in a more general expression of type $u=\gamma f(\alpha, \beta)$, where $f$ is an arbitrary function and $u$ satisfies the wave equation, the problem can be solved as follows:

It is easily seen that the function $f$ must satisfy the differential equation

$$
\left(\frac{\partial f}{\partial x}\right)^{2}+\left(\frac{\partial f}{\partial y}\right)^{2}+\left(\frac{\partial f}{\partial z}\right)^{2}=\frac{1}{c^{2}}\left(\frac{\partial f}{\partial t}\right)^{2}
$$

which gives rise to three equations connecting $\alpha$ and $\beta$ when the coefficients of

$$
\left(\frac{\partial f}{\partial \alpha}\right)^{2}, \frac{\partial f}{\partial \alpha} \frac{\partial f}{\partial \beta}, \text { and }\left(\frac{\partial f}{\partial \beta}\right)^{2}
$$

are equated to zero.

Now let $x, y, \alpha, \beta$ be taken as new independent variables; the preceding equation then takes the form

$$
K^{-2}\left[A X^{2}-2 H X Y+B Y^{2}\right]=0,
$$

where

$$
\begin{aligned}
& A=\left(\frac{\partial t}{\partial x}\right)^{2}+\left(\frac{\partial t}{\partial y}\right)^{2}-\frac{1}{c^{2}}, \quad B=\left(\frac{\partial z}{\partial x}\right)^{2}+\left(\frac{\partial z}{\partial y}\right)^{2}+1, \\
& H=\frac{\partial z}{\partial x} \frac{\partial t}{\partial x}+\frac{\partial z}{\partial y} \frac{\partial t}{\partial y}, \quad K=\frac{\partial(z, t)}{\partial(\alpha, \beta)}, \\
& X=\frac{\partial(f, z)}{\partial(\alpha, \beta)}, \quad Y=\frac{\partial(f, t)}{\partial(\alpha, \beta)} \text {. }
\end{aligned}
$$

${ }^{*} E$, p. 123. 
Equating to zero the coefficients of

$$
\left(\frac{\partial f}{\partial \alpha}\right)^{2}, \quad \frac{\partial f}{\partial \alpha} \frac{\partial f}{\partial \beta},\left(\frac{\partial f}{\partial \beta}\right)^{2},
$$

we obtain three linear equations connecting $A, B, H$. The determinant of the coefficients is easily found to be $K^{-3}$, and this is zero only when there is a relation between $\alpha, \beta, x, y$. Ignoring this trivial case, we may conclude from (6) that $A=0, B=0, H=0$. Now in virtue of the last equation we may write

$$
\frac{\partial t}{\partial x}=\lambda \frac{\partial z}{\partial y}, \quad \frac{\partial t}{\partial y}=-\lambda \frac{\partial z}{\partial x_{i}^{\prime}}
$$

where $\lambda$ is some function of $x, y, \alpha, \beta$. Substituting in the equation $A=0$, we find that $c^{2} \lambda^{2}+1=0$, hence

$$
c \frac{\partial t}{\partial x}=i \frac{\partial z}{\partial y}, \quad c \frac{\partial t}{\partial y}=-i \frac{\partial z}{\partial x} .
$$

These equations imply that

$$
z-c t=F(x+i y), \quad z+c t=G(x-i y),
$$

where $F$ and $G$ are functions which depend also on $\alpha$ and $\beta$. Substituting these expressions in the equation $B=0$, we find that

$$
F^{\prime}(x+i y) G^{\prime}(x-i y)+1=0 .
$$

Hence we may put

$$
z-c t=\phi+\theta(x+i y), \quad z+c t=\psi-\frac{1}{\theta}(x-i y),
$$

where $\theta, \phi, \psi$ are arbitrary functions of $\alpha$ and $\beta$. These equations may be used to define $\alpha$ and $\beta$ as functions of $x, y, z, t$. It has already been shown* that when $\alpha$ and $\beta$ are defined by equations of this type a solution of the wave equation (4) may be obtained by writing $u=\gamma f(\alpha, \beta)$ where $\gamma$ is one of the Jacobians of type $\partial(\alpha, \beta) / \partial(y, z)$. The preceding analysis indicates that all the wave functions of this type may be obtained by the method just described; to complete the proof we must show that the ratio of two suitable values of $\gamma$ depends only on $\alpha$ and $\beta$.

* Messenger of Mathematics, March, 1914, p. 164; E, p. 134. 
Let us suppose that $\gamma f(\alpha, \beta)$ and $\gamma^{\prime} F(\alpha, \beta)$ satisfy the wave equation, where $f$ and $F$ are arbitrary; then it is easy to deduce that

$$
\begin{gathered}
\frac{\partial w}{\partial x} \frac{\partial \alpha}{\partial x}+\frac{\partial w}{\partial y} \frac{\partial \alpha}{\partial y}+\frac{\partial w}{\partial z} \frac{\partial \alpha}{\partial z}=\frac{1}{c^{2}} \frac{\partial w}{\partial t} \frac{\partial \alpha}{\partial t}, \\
\frac{\partial w}{\partial x} \frac{\partial \beta}{\partial x}+\frac{\partial w}{\partial y} \frac{\partial \beta}{\partial y}+\frac{\partial w}{\partial z} \frac{\partial \beta}{\partial z}=\frac{1}{c^{2}} \frac{\partial w}{\partial t} \frac{\partial \beta}{\partial t},
\end{gathered}
$$

where $w=\gamma^{\prime} / \gamma$. These equations imply that

$$
\frac{\partial w}{\partial x}=P \frac{\partial \alpha}{\partial x}+Q \frac{\partial \beta}{\partial x}, \quad \frac{\partial w}{\partial y}=P \frac{\partial \alpha}{\partial y}+Q \frac{\partial \beta}{\partial y}, \quad \text { etc. } ;
$$

for $w=\alpha$ and $w=\beta$ are evidently particular solutions of the preceding equations. We now have $d w=P d \alpha+Q d \beta$, whence it follows that $w$ is a function of $\alpha$ and $\beta$.

I have shown elsewhere* that if $\alpha$ and $\beta$ are defined by the equations

$$
\begin{aligned}
(x-\xi)^{2}+(y-\eta)^{2}+(z-\zeta)^{2} & =c^{2}(t-\tau)^{2}, \\
l(x-\xi)+m(y-\eta)+n(z-\zeta) & =c p(t-\tau),
\end{aligned}
$$

where $\xi, \eta, \zeta, \tau, l, m, n, p$ are functions of $\alpha$ and $\beta$ subject to the relation $l^{2}+m^{2}+n^{2}=p^{2}$, then wave functions of type $\gamma f(\alpha, \beta)$ exist. It is easily seen that equations (8) are equivalent to (7) if

$$
\begin{gathered}
\phi=\zeta-c \tau-\theta(\xi+i \eta), \quad \psi=\zeta+c \tau+\frac{1}{\theta}(\xi-i \eta), \\
\theta=-\frac{l-i m}{n+p}=\frac{z-\zeta-c(t-\tau)}{x-\xi+i(y-\eta)} .
\end{gathered}
$$

An æthereal field may be specified with the aid of the functions $\alpha, \beta$ by defining the components of $M$ by equations of type $\dagger$

$$
M_{x}=f(\alpha, \beta) \frac{\partial(\alpha, \beta)}{\partial(y, z)}=\frac{i}{c} f(\alpha, \beta) \frac{\partial(\alpha, \beta)}{\partial(x, t)} .
$$

\section{§ 5. Model of an Element of the Ether.}

Let us now consider the case when $\tau=\alpha$ and $\xi, \eta, \zeta$ are functions of $\alpha$ only; then as Liénard has shown, the first of

${ }^{*} E$, Ch. 8

$\dagger E$, pp. $12,122$. 
equations (8) will define just one real value of $\alpha$ if the inequalities

$$
\tau \leq t, \quad \xi^{\prime 2}+{\eta^{\prime}}^{2}+{\zeta^{\prime}}^{2}<c^{2}, \quad\left(\xi^{\prime}=d \xi / d \tau\right),
$$

are added. If, moreover, $l, m, n, p$ are linear functions of $\beta$ the second of equations (8) will define just one value of $\beta$ and this will generally be a complex function of $x, y, z, t$. Let us choose the function $f(\alpha, \beta)$ in formulas (9) so that $f^{-1}=\beta-\beta_{0}$, where $\beta_{0}$ is an arbitrarily chosen complex quantity;* the components of $M$ will then generally be infinite at points on the moving curve $\beta=\beta_{0}$.

Now when $\beta=\beta_{0}$ and $\alpha$ is kept fixed, $\theta$ has a definite complex value and so it follows that the line joining the points $(x, y, z, t),(\xi, \eta, \zeta, \tau)$ is fixed in direction. Taking into account the first of equations (8) we see that the possible positions of $(x, y, z, t)$ are those of a point which starts at $(\xi, \eta, \zeta, \tau)$ and travels along a straight line with the velocity of light. By giving different real values to $\alpha$ we obtain a series of such points which form at any instant a singular curve of the æthereal field specified by (9). This singular curve is attached to the moving point $(\xi, \eta, \zeta, \tau)$. If we give different complex values to $\beta_{0}$, a series of such curves is obtained, there being one for each value of $\beta_{0}$.

Let us now see if it is possible to choose the arbitrary functions at our disposal in such a way that the singular curve of an æthereal field of the type just considered is always a line of electric force in an electromagnetic field having the moving point $(\xi, \eta, \zeta, \tau)$ as simple singularity.

Liénard has shown that an electromagnetic field with $(\xi, \eta, \zeta, \tau)$ as a moving point charge may be derived from the potentials

$$
A_{x}=\xi^{\prime} / \nu, \quad A_{y}=\eta^{\prime} / \nu, \quad A_{z}=\zeta^{\prime} / \nu, \quad \Phi=c / \nu,
$$

where

$$
\nu=\xi^{\prime}(x-\xi)+\eta^{\prime}(y-\eta)+\zeta^{\prime}(z-\zeta)-c^{2}(t-\tau) .
$$

Writing

$$
\begin{gathered}
x-\xi=c l_{0}(t-\tau), \quad y-\eta=c m_{0}(t-\tau), \\
x-\zeta=c n_{0}(t-\tau), \\
c-l_{0} \xi^{\prime}-m_{0} \eta^{\prime}-\eta_{0} \zeta^{\prime}=\lambda, \quad c^{2}-\xi^{2}-\eta^{\prime 2}-\zeta^{\prime 2}=\mu,
\end{gathered}
$$

* It may be a function of $\alpha$ but not of $\beta$. 
we find on calculating the components of $E$ by means of (2) that

$$
\begin{aligned}
E_{x}=\frac{1}{c \nu^{2}} \frac{\partial \tau}{\partial t}\left[\mu\left(\xi^{\prime}-c l_{0}\right)\right. & +c(t-\tau)\left\{\lambda \xi^{\prime \prime}\right. \\
& \left.\left.+\left(\xi^{\prime}-c l_{0}\right)\left(l_{0} \xi^{\prime \prime}+m_{0} \eta^{\prime \prime}+n_{0} \xi^{\prime \prime}\right)\right\}\right] .
\end{aligned}
$$

Now let $l_{0}, m_{0}, n_{0}$ be regarded as functions of $\tau$ and let us suppose that particles are projected from the different positions of the singularity $(\xi, \eta, \zeta, \tau)$ in directions specified by the direction cosines $\left(l_{0}, m_{0}, n_{0}\right)$, and that they travel along straight lines with the velocity of light. If $(x, y, z),(x+d x$, $y+d y, z+d z)$ are the coordinates at time $t$ of two particles that were projected at times $\tau, \tau+d \tau$, we find that

$$
\frac{d x}{d \tau}=\xi^{\prime}-c l_{0}+c(t-\tau) \frac{d l_{0}}{d \tau} .
$$

Comparing this with the expression for $E_{x}$, we see that the line joining two consecutive particles coincides in direction with a portion of a line of electric intensity at each time $t$, if the derivatives of $l_{0}, m_{0}, n_{0}$ are given by equations of type

$$
\mu \frac{d l_{0}}{d \tau}=\lambda \xi^{\prime \prime}+\left(\xi^{\prime}-c l_{0}\right)\left(l_{0} \xi^{\prime \prime}+m_{0} \eta^{\prime \prime}+n_{0} \xi^{\prime \prime}\right) .
$$

If this condition is satisfied, the series of projected particles will form at each instant $t$ a line of electric force in the electromagnetic field with the singularity $(\xi, \eta, \zeta, \tau)$. By taking different initial directions for $\left(l_{0}, m_{0}, n_{0}\right)$ we may obtain in this way all the lines of electric force.

Since the functions $l, m, n, p$ are arbitrary as far as their dependence on $\alpha$ is concerned, we may evidently choose them so that the direction given by the equation $\beta=\beta_{0}$ coincides at each instant $\tau$ with the direction specified by the functions $\left(l_{0}, m_{0}, n_{0}\right)$. This means that we can choose a system of æthereal fields in such a way that their singular curves coincide at each instant with the lines of electric force in an electromagnetic field having $(\xi, \eta, \zeta, \tau)$ as simple singularity.

As regards the conditions of conjugacy, we have already proved that the electromagnetic field specified by the potentials (10) is conjugate to any field of type (9) in which $\alpha, \beta$ are the special functions considered in this paragraph.* We

* $E$, pp. 126, 133. 
have also proved that a field of type (9) is self-conjugate* and is such that the flow of energy (Poynting's vector) is along the radius from $(\xi, \eta, \zeta, \tau)$ to $(x, y, z, t)$, hence it follows that the different æthereal fields given by (9) form a mutually conjugate system.

There are still many questions to be answered before the present theory can be accepted. If the æther is regarded as built up of elements a law describing the mutual action of two elements is needed. The nature of the macroscopic equations satisfied by the vectors $(E, H)$ in a region occupied by an enormous aggregate of singular curves of æthereal fields has not been ascertained. The field obtained by superposing all the æthereal fields is probably different in character from an ordinary electromagnetic field; it may possibly have some connection with the phenomena of gravitation.

JoHNS HopkINS UNIVERSITY, BALTIMORE, October 27, 1914.

\section{SHORTER NOTICES.}

Handbuch des mathematischen Unterrichts. Von W. KILLING und H. Hovestadt. Band II. Leipzig and Berlin, Teubner, 1913. $\mathrm{x}+472 \mathrm{pp}$.

THE general character of this work was discussed by the reviewer in the BuLLETIN, volume 17, No. 5. The vigor which characterized the first volume is unabated in the present one, which is devoted to trigonometry. One third of the volume is devoted to plane trigonometry, an equal amount to solid geometry or stereometry in which plane trigonometry is used freely in dealing with space problems, and the remainder to spherical trigonometry. There is sharp criticism of the ordinary text-books on trigonometry, in which the authors deplore the tendency to limit the field to goniometry and the solution of triangles with and without logarithms. Nor are they satisfied with a criticism of the content, for the methods of proof come under fire as well. As an illustration of the carefulness of their investigation, four proofs of the addition

\footnotetext{
*E, p. 12; Phil. Mag., Jan., 1914.
} 